\title{
Spectral extrema for graphs: the Zarankiewicz problem
}

\author{
László Babai* \\ Barry Guiduli ${ }^{\dagger}$
}

Submitted: Jul 12, 2007; Accepted: Sep 21, 2009; Published: Sep 25, 2009

\begin{abstract}
Let $G$ be a graph on $n$ vertices with spectral radius $\lambda$ (this is the largest eigenvalue of the adjacency matrix of $G$ ). We show that if $G$ does not contain the complete bipartite graph $K_{t, s}$ as a subgraph, where $2 \leqslant t \leqslant s$, then

$$
\lambda \leqslant\left((s-1)^{1 / t}+o(1)\right) n^{1-1 / t}
$$

for fixed $t$ and $s$ while $n \rightarrow \infty$. Asymptotically, this bound matches the KöváriTurán-Sós upper bound on the average degree of $G$ (the Zarankiewicz problem).
\end{abstract}

\section{Introduction}

The Zarankiewicz problem [16] is one of the classical and still largely open problems in extremal graph theory. The problem asks for the maximum number $m$ of edges in a graph on $n$ vertices which does not contain the complete bipartite graph $K_{t, s}$. We will assume that $2 \leqslant t \leqslant s$ throughout the paper. We formulate the results in terms of the average degree $2 m / n$. The following upper bound was given in 1954 by Kővári, Sós, and Turán [1] (see also [2]).

Theorem 1 (Kövári-Sós-Turán). Let $G$ be a graph on $n$ vertices with $m$ edges. If $G$ does not contain $K_{t, s}$ as a subgraph then the average degree in $G$ is

$$
2 m / n \leqslant(s-1)^{1 / t} n^{1-1 / t}+t-1 .
$$

In 1996, Füredi [7] improved the asymptotic coefficient $(s-1)^{1 / t}$ to $(s-t+1)^{1 / t}$.

Theorem 2 (Füredi). Under the conditions of Theorem 1 ,

$$
2 m / n \leqslant(s-t+1)^{1 / t} n^{1-1 / t}+t n^{1-2 / t}+t .
$$

\footnotetext{
*University of Chicago. E-mail: laci@cs.uchicago.edu
}

${ }^{\dagger}$ E-mail: barry@guiduli.com 
The order of magnitude $n^{1-1 / t}$ is conjectured best possible, but this has only been proven for special cases: For $t=2$, it was proved by E. Klein (as reported by P. Erdős in [5]). W. G. Brown [4] showed it for $t=3$, and more recently, Kollár, Rónyai, and Szabó [10] showed it for all $(t, s)$ satisfying $s \geqslant t !+1$ (improved to $s \geqslant(t-1)$ ! in [1]). For the case $t=2$ even the coefficient of the leading $n^{1-1 / t}$ term given by Kövári, Sós, and Turán has been shown to be best possible (E. Klein for $s=2$, Z. Füredi 6 for all $s$ ). For $t=s=3$, Füredi's upper bound (11) and Brown's lower bound [4] are asymptotically equal, showing the optimality of their coefficient.

The spectral radius $\lambda$ of $G$ is the largest eigenvalue of the adjacency matrix of $G$. The spectral radius satisfies the inequality $2 m / n \leqslant \lambda$ and in this sense, bounds on the spectral radius generalize bounds on the number of edges. Our main result establishes asymptotically the Kővári-Sós-Turán upper bound on $\lambda$.

Theorem 3. Let $G$ be a graph on $n$ vertices with spectral radius $\lambda$. If $G$ does not contain $K_{t, s}$ as a subgraph, where $2 \leqslant t \leqslant s$, then

$$
\lambda \leqslant\left((s-1)^{1 / t}+o(1)\right) n^{1-1 / t}
$$

where the o(1) refers to fixed $t$ and $s$ while $n \rightarrow \infty$.

By Füredi's result [6] it follows that in the case $t=2$, our result is asymptotically best possible (including the constant $(s-1)^{1 / 2}$ ) for all $s$.

Although our result implies the asymptotic bound inferred from Theorem 11 this paper does not provide an alternative proof of that result because our bound for a lower order term relies on Theorem 1. On the other hand, our bound for the main term relies on a direct argument independent of Theorem 11. therefore we will not gain by replacing the KST bound by Füredi's bound (Theorem 2) in our argument.

Problem 1. 1 Prove that under the conditions of Theorem 3 ,

$$
\lambda \leqslant\left((s-t+1)^{1 / t}+o(1)\right) n^{1-1 / t}
$$

i. e., establish the Füredi bound on $\lambda$.

We will prove Theorem [3] in two parts. In Section [3] we consider the case $2=t \leqslant s$, and in Section 4 we consider the case $3 \leqslant t \leqslant s$.

\section{Preliminaries}

The complete bipartite graph $K_{t, s}$ is the graph whose vertex set is the union of the disjoint sets $T$ and $S,|T|=t$ and $|S|=s$, and whose edges are all pairs $\{x, y\}$, for $x \in T$ and $y \in S$.

\footnotetext{
${ }^{1}$ (Added in 2009) Most recently, Vlado Nikiforov solved this problem [15].
} 
Throughout the paper, $G$ is a graph on vertex set $V=\{1,2, \ldots, n\}$. The $n \times n$ adjacency matrix $A=\left(a_{i j}\right)$ of $G$ is defined by setting $a_{i j}=1$ if $i$ and $j$ are adjacent, 0 otherwise. The largest eigenvalue of $A$ is denoted $\lambda$ and is called the spectral radius of $G$. Further, let $\mathbf{x}=\left\{x_{1}, \ldots, x_{n}\right\}$ be a nonnegative eigenvector for $\lambda$. (We refer the reader to Chapter 11 of Lovász's book 13 for background on eigenvalues of graphs.)

We use the following additional notation for any subset $W \subseteq\{1, \ldots, n\}$ :

$$
\begin{aligned}
S_{W} & :=\sum_{i \in W} x_{i}, \\
S_{W}^{r} & :=\left(\sum_{i \in W} x_{i}\right)^{r}, \\
P_{W}(r) & :=\sum_{i \in W} x_{i}^{r} .
\end{aligned}
$$

Fact 1. For $r$ a positive integer and $W \subseteq V$ we have

$$
\left(\sum_{i \in W} x_{i}\right)^{r} \leqslant|W|^{r-1} \sum_{i \in W} x_{i}^{r}, \quad \text { i.e., } \quad S_{W}^{r} \leqslant|W|^{r-1} P_{W}(r) .
$$

Fact 2 (Adolf Szücs (see [12])). Let $a_{1} \leqslant \cdots \leqslant a_{n}$ and $b_{1} \leqslant \cdots \leqslant b_{n}$ be nonnegative real numbers. Then for every permutation $\sigma$ on $\{1, \ldots, n\}$,

$$
\sum_{i} a_{i} b_{i} \geqslant \sum_{i} a_{i} b_{\sigma(i)}
$$

The following fact is a consequence of the previous one.

Fact 3. Let $\mathbf{x}$ be a nonnegative vector, and $a$ and $b$ nonnegative real numbers. Then

$$
\left(\sum_{i \in W} x_{i}^{a}\right)\left(\sum_{i \in W} x_{i}^{b}\right) \leqslant|W| \sum_{i \in W} x_{i}^{a+b}, \quad \text { i.e., } \quad P_{W}(a) P_{W}(b) \leqslant|W| P_{W}(a+b) .
$$

\section{$3 \quad K_{2, s}$-free graphs}

In this section we prove the following bound.

Theorem 4. Let $G$ be a graph on $n$ vertices with spectral radius $\lambda$. If $G$ does not contain $K_{2, s}$ as a subgraph where $2 \leqslant s$ then

$$
\lambda \leqslant(s-1)^{1 / 2} n^{1 / 2}+O\left((n s)^{1 / 4}\right) .
$$

We will actually prove that

$$
\lambda<(s-1)^{1 / 2} n^{1 / 2}+2^{-3 / 4}(s-1)^{1 / 4} n^{1 / 4}+2^{-1 / 2} .
$$


Proof: Recall that $\mathbf{x}=\left(x_{1}, \ldots, x_{n}\right)$ is a nonnegative eigenvector for $\lambda$. The Theorem follows by bounding the expression $\lambda^{2} \sum_{i} x_{i}^{2}$. We may rewrite this as:

$$
\lambda^{2} \sum_{i} x_{i}^{2}=\sum_{i}\left(\lambda x_{i}\right)^{2}=\sum_{i}\left(\sum_{j \sim i} x_{j}\right)^{2}=\sum_{i} d_{i} x_{i}^{2}+\sum_{i \neq j} d_{i j} x_{i} x_{j}
$$

where $d_{i}$ is the degree of vertex $i$ and $d_{i j}$ is the number of common neighbors of $i$ and $j$. (The tilde denotes adjacency.) To simplify notation, we will use $P=\sum_{i} x_{i}^{2}$ and $P_{X}=P_{X}(2)=\sum_{i \in X} x_{i}^{2}$. We get a reasonable bound on $\lambda^{2} P$ using only the facts that $d_{i}<n$ and $d_{i j}<s-1$, by virtue of the assumption that $G$ does not contain $K_{2, s}$. Using Fact 3 we may immediately conclude:

$$
\lambda^{2} P=\sum_{i} d_{i} x_{i}^{2}+\sum_{i \neq j} d_{i j} x_{i} x_{j}<n P+(s-1) n P=s n P,
$$

implying that $\lambda<s^{1 / 2} n^{1 / 2}$. To reduce the constant to the optimal $(s-1)^{1 / 2}$, we need to examine the two terms more carefully.

We will partition the vertices of $G$ into a set $U$ of vertices of "high" degree and a set of vertices of "low" degree, and split our sums accordingly.

Let $D=(2(s-1) n)^{1 / 2}$. Set $U=\left\{i \in V: d_{i}>D\right\}$ and $W=V \backslash U$. With this notation we have

$$
\sum_{i} d_{i} x_{i}^{2} \leqslant n P_{U}+D P_{W} \leqslant n P_{U}+D P .
$$

We claim that $U$ is smaller than would be predicted by counting edges.

Claim 1. $|U| \leqslant(2 n /(s-1))^{1 / 2}=D /(s-1)$.

To prove the Claim, let $u=|U|$ and assume that $G$ is labeled so that $U=\{1, \ldots, u\}$. Let $N_{i} \subset V$ be the set of neighbors of $i$. Then $\left|N_{i} \cap N_{j}\right| \leqslant s-1$ whenever $i \neq j$. Thus the union of any $k$ of the $N_{i}$ must contain at least $f(k):=D+(D-(s-1))+$ $\cdots+(D-(k-1)(s-1))$ elements. Therefore $f(k) \leqslant n$. Now the quadratic function $f(x)=(x / 2)(2 D+s-1-x)$ takes its maximum at $x_{0}=D+(s-1) / 2$ so it is increasing up to $x_{0}$. Therefore to verify that $k \leqslant D /(s-1)$, we only need to show that $f(D /(s-1))>n$, i. e., $D\left(2 D+s-1-(D /(s-1))>2 n(s-1)=D^{2}\right.$. This reduces to $D(s-2)+(s-1)^{2}>0$, which is true.

Now, using the fact that $d_{i j} \leqslant s-1$, we have

$$
\sum_{i, j} d_{i j} x_{i} x_{j} \leqslant(s-1) \sum_{i, j} x_{i} x_{j}
$$

and after factoring out $(s-1)$ from the the second summation on the right-hand side 
of (3), we may bound it as follows:

$$
\begin{aligned}
\sum_{i \neq j} x_{i} x_{j} & =\sum_{i, j \in U, i \neq j} x_{i} x_{j}+\sum_{i, j \in W, i \neq j} x_{i} x_{j}+2 S_{U} S_{W} \\
& \leqslant(u-1) P_{U}+(|W|-1) P_{W}+2 S_{U} S_{W} \\
& <u P_{U}+n P_{W}+2 \sqrt{u n P_{U} P_{W}} \\
& \leqslant u P+n P_{W}+\sqrt{u n} P .
\end{aligned}
$$

Combining this last inequality with inequalities (15), (6), and the first equation in (41), and observing that

$$
n P_{U}+(s-1) n P_{W} \leqslant(s-1) n P
$$

we obtain

$$
\begin{aligned}
\lambda^{2} & \leqslant \frac{1}{P}\left(n P_{U}+D P+(s-1) u P+(s-1) n P_{W}\right)+(s-1) \sqrt{u n} \\
& \leqslant(s-1) n+D+(s-1) u+(s-1) \sqrt{u n}
\end{aligned}
$$

and using $u \leqslant D /(s-1)$ (Claim 1),

$$
\lambda^{2} \leqslant(s-1) n+2 D+\sqrt{n D(s-1)} .
$$

Substituting in the value of $D$, we arrive at our conclusion:

$$
\begin{aligned}
\lambda^{2} & \leqslant(s-1) n+2^{1 / 4}(s-1)^{3 / 4} n^{3 / 4}+2^{3 / 2}(s-1)^{1 / 2} n^{1 / 2} \\
& \leqslant\left((s-1)^{1 / 2} n^{1 / 2}+2^{-3 / 4}(s-1)^{1 / 4} n^{1 / 4}+2^{-1 / 2}\right)^{2} .
\end{aligned}
$$

This completes the proof of inequality (2).

\section{$4 \quad K_{t, s}$-free graphs}

In this section we prove the following result which makes the error-term in Theorem 3 explicit.

Theorem 5. Let $G$ be a graph on $n$ vertices with spectral radius $\lambda$, and let $3 \leqslant t \leqslant s \leqslant n$. If $G$ does not contain $K_{t, s}$ as a subgraph, then

$$
\lambda \leqslant(s-1)^{1 / t} n^{1-1 / t}+c(t, s) n^{1-\frac{1}{t}-\frac{t-2}{t(t-1)}} .
$$

where $c(t, s)<3 t / 2$ for all $t$ and $s$.

We prove that $c(t, s) \leqslant(3 / 2)(t-1)(s-1)^{-\left(1-\frac{2}{t}+\frac{1}{t(t-1)}\right)}$.

We need the Kövári-Sós-Turán upper bound on the maximum number of edges in a $K_{t, s}$-free graph to bound the number of vertices of large degree. 
Lemma 1. Let $G$ be a graph on $n$ vertices not containing $K_{t, s}$. Then $G$ has fewer than

$$
(s-1)^{\frac{1}{t(t-1)}} n^{1-\frac{1}{t(t-1)}}(1+\epsilon(t, s, n))
$$

vertices of degree at least

$$
(s-1)^{\frac{t-2}{t(t-1)}} n^{1-\frac{t-2}{t(t-1)}}
$$

where $0<\epsilon(t, s, n)<t n^{-1+(1 / t)}$.

Proof: If $G$ had more than this many vertices of this degree, then it would have more edges than allowed by Theorem 1 .

Proof of Theorem 5: We consider the $t$-th power of $\lambda$ :

$$
\begin{aligned}
\lambda^{t} P_{V}(t) & =\sum_{i=1}^{n}\left(\lambda x_{i}\right)^{t}=\sum_{i=1}^{n}\left(\sum_{j \sim i} x_{j}\right)^{t} \\
& =\sum_{i_{1}=1}^{n} \cdots \sum_{i_{t}=1}^{n} d\left(i_{1}, \ldots, i_{t}\right) x_{i_{1}} \cdots x_{i_{t}}
\end{aligned}
$$

where $d\left(i_{1}, \ldots, i_{t}\right)$ is the number of common neighbors of the $t$ (not necessarily distinct) vertices $i_{1}, \ldots, i_{t}$. If the vertices are distinct, this is at most $s-1$, otherwise it it is less than $n$.

We break the sum up into two parts. The first part is all summands for which the $t$-tuple consists of $t$ distinct vertices, and we call this $A$. This sum may be bounded above by

$$
A \leqslant(s-1)\left(\sum_{i=1}^{n} x_{i}\right)^{t} \leqslant(s-1) n^{t-1} P_{V}(t),
$$

by Fact 1 . The part which remains we call $B$. This may be bounded above by

$$
B \leqslant\left(\begin{array}{l}
t \\
2
\end{array}\right) \sum_{i_{1}=1}^{n} \cdots \sum_{i_{t-2}=1}^{n} \sum_{j=1}^{n} d\left(i_{1}, \ldots, i_{t-2}, j\right) x_{i_{1}} \cdots x_{i_{t-2}} x_{j}^{2} .
$$

Indeed, if the components of a $t$-tuple are not distinct, equality will occur between at least one of the $\left(\begin{array}{l}t \\ 2\end{array}\right)$ pairs of positions. We will show that the right-hand side of (9) is $o\left(n^{t-1}\right)$, thus completing the proof.

Let $U$ be the set of vertices of degree at least

$$
D=(s-1)^{\frac{t-2}{t(t-1)}} n^{1-\frac{t-2}{t(t-1)}},
$$

and let $W$ be the set of remaining vertices. By Lemma 1, we see that

$$
|U| \leqslant(s-1)^{\frac{1}{t(t-1)}} n^{1-\frac{1}{t(t-1)}}(1+\epsilon(t, n, s)) .
$$


We now split each of the sums into parts corresponding to $U$ and $W$, and then use the fact that if at least one vertex is from $W$, then the number of common neighbors can be at most $D$. Hence the sum is bounded above by

$$
\sum_{i_{1}=1}^{n} \cdots \sum_{i_{t-2}=1}^{n} d\left(i_{1}, \ldots, i_{t-2}, j\right) x_{i_{1}} \cdots x_{i_{t-2}} \sum_{j=1}^{n} x_{j}^{2} \leqslant D S_{V}^{t-2} P_{V}(2)+n S_{U}^{t-2} P_{U}(2) .
$$

Combining Facts 1 and 3 we obtain $S_{V}^{t-2} P_{V}(2) \leqslant n^{t-2} P_{V}(t)$, hence the first term above is bounded by

$$
D S_{V}^{t-2} P_{V}(2) \leqslant D n^{t-2} P_{V}(t)=(s-1)^{\frac{t-2}{t(t-1)}} n^{t-1-\frac{t-2}{t(t-1)}} P_{V}(t)
$$

The second term satisfies nearly the same bound (a factor of 2 appears only as a specific upper bound on $\left.(1+\epsilon)^{t-2}=1+o(1)\right)$,

$$
n S_{U}^{t-2} P_{U}(2) \leqslant n|U|^{t-2} P_{U}(t) \leqslant 2(s-1)^{\frac{t-2}{t(t-1)}} n^{t-1-\frac{t-2}{t(t-1)}} P_{V}(t) .
$$

Thus

$$
\lambda^{t} \leqslant(s-1) n^{t-1}\left(1+3\left(\begin{array}{l}
t \\
2
\end{array}\right)(s-1)^{-1+\frac{t-2}{t(t-1)}} n^{-\frac{t-2}{t(t-1)}}\right) .
$$

Noting that $(1+x)^{1 / t} \leqslant 1+x / t(x>0, t>1)$, we conclude that

$$
\lambda \leqslant(s-1)^{1 / t} n^{1-1 / t}\left(1+(3 / 2)(t-1)(s-1)^{-1+\frac{t-2}{t(t-1)}} n^{-\frac{t-2}{t(t-1)}}\right),
$$

verifying (17). This completes the proof of Theorem [5.

\section{History.}

This work started in 1996 as part of a bigger project on spectral extrema for graphs while the junior author worked on his Ph. D. thesis at the University of Chicago under the supervision of the senior author. The paper was completed in its present form (apart from minor recent polishing) on February 8, 1998. Subsequently the manuscript fell victim to the senior author's personal odyssey and the junior author's move away from academia. The junior author now works for an investment bank in New York City.

Some published (cf. 9]) and numerous unpublished results of this project can be found in the junior author's thesis [8]. The main result of the present paper appears there as Theorem 4.17, with an outline of the proof given here in full detail. During the intervening years, unaware of [8], Vladimir Nikiforov, in part with Béla Bollobás, embarked on a similar project (cf. [14, 3] and many other papers accessible on Vlado's home page). In 2007, Vlado discovered the abstract of a talk given by the junior author a decade earlier in France during his postdoc year at Laboratoire Leibniz, IMAG, Grenoble, where several of the results of his thesis were announced (cf. links at [8]). We are grateful 
to Vlado for having contacted us at that point as well as for his encouragment and critical comments on various results in [8].

Acknowledgment. The authors wish to thank the anonymous referees for their comments and in particular for pointing out Füredi's paper [7].

\section{References}

[1] Noga Alon, Lajos Rónyai, Tibor Szabó: Norm graphs: variations and applications. J. Combinat. Theory, Ser. B, 76 (1999) pp. 280-290.

[2] BÉla Bollobás: Extremal Graph Theory. Academic Press, London, 1978.

[3] Béla Bollobás, Vladimir Nikiforov: Cliques and the Spectral Radius. J. Combinat. Theory, Ser. B 97 (2007), 859-865.

[4] W. G. Brown: On graphs that do not contain a Thomsen graph. Canad. Math. Bull. 9 (1966), pp. 281-289.

[5] PÁl ERdős: On sequences of integers no one of which divides the product of two others and on some related problems. Isvestia Nauchno-Issl. Inst. Mat. i Meh. Tomsk 2 (1938), pp. 74-82.

[6] Zoltán Füredi: New asymptotics for bipartite Turán numbers. J. Combinat. Theory, Ser. A, 75 (1996), pp. 141-144.

[7] Zoltán FÜRedi: An upper bound on Zarankiewicz' problem. Combinatorics, Probability and Computing, 5 (1996), pp. 29-33.

[8] Barry Guiduli: Spectral Extrema for Graphs. Ph.D. Thesis, Department of Mathematics, University of Chicago, December 1996. URL: http://people.cs.uchicago.edu/ laci/students

[9] Barry Guiduli: The Structure of Trivalent Graphs with Minimal Eigenvalue Gap. J. Algebraic Combinatorics 6/4 (1997), 321-329.

[10] János Kollár, Lajos Rónyai, Tibor Szabó: Norm graphs and bipartite Turán numbers. Combinatorica, 16 (1996) pp. 399-406.

[11] Tamás Kövári, Vera T. Sós, PÁl Turán: On a problem of K. Zarankiewicz, Colloq. Math. 3 (1954), pp. 50-57.

[12] József Kürschák, György Hajós, Gyula Neukomm, János Surányi, Eds.: Hungarian Problem Book. Random House, New York 1963.

[13] LÁszló Lovász: Combinatorial Problems and Exercises. Akadémiai Kiadó, Budapest, 1993.

[14] Vladimir Nikiforov: Some inequalities for the largest eigenvalue of a graph. Combinatorics, Probability and Computing 11 (2002), 179-189.

[15] Vladimir Nikiforov: A contribution to the Zarankiewicz problem. Manuscript, 2009. https://umdrive.memphis.edu/vnikifrv/public/list4.htm

[16] Kazimierz Zarankiewicz: Problem of P101, Colloq. Math., 2 (1951), p. 301. 